\title{
Unique CYP3A4 genetic variant in Brazilian tuberculosis patients with/without HIV
}

\author{
ANDRÉ L. JEOVANIO-SILVA ${ }^{1}$, THAÍS P. MONTEIRO ${ }^{1}$, KÊNIA B. EL-JAICK ${ }^{1}$, \\ PEDRO E.A.A. DO BRASIL ${ }^{2}$, VALÉRIA C. ROLLA ${ }^{3}$ and LIANE DE CASTRO ${ }^{1}$ \\ ${ }^{1}$ Laboratório de Biomarcadores e Hepatotoxicidade; ${ }^{2}$ Laboratório de Pesquisa Clínica em Doença de Chagas; \\ ${ }^{3}$ Laboratório de Pesquisa Clínica em Micobacterioses, Instituto de Pesquisa Clínica Evandro Chagas - \\ Fundação Oswaldo Cruz (IPEC/FIOCRUZ), Manguinhos, Rio de Janeiro 21040-360, RJ, Brazil
}

Received April 15,2011; Accepted August 17, 2011

DOI: $10.3892 / \mathrm{mmr} .2011 .602$

\begin{abstract}
CYP3A4 is involved in tuberculosis (TB) and human immunodeficiency virus (HIV) drug metabolism. Transcriptional activation by rifampicin involves the CYP3A4 gene 5'-upstream region. Consequently, variation may interfere with transcription and enzymatic activity and even drug response. However, genetic polymorphisms and distribution of CYP3A4 allelic frequencies in individuals from Rio de Janeiro remain unknown. The aim of this study was to conduct research into sequencing the CYP3A4 5'-upstream region in Brazilian patients with and without HIV. This follow-up study involved 106 individuals undergoing treatment for TB and/ or HIV. The CYP3A4 5'-upstream region was analyzed using PCR, sequencing and clinical data. Male patients revealed a higher HIV frequency ( $\mathrm{p}=0.021)$. The TB forms observed were pulmonary $(48.1 \%)$, extrapulmonary $(22.64 \%)$ and disseminated $(27.36 \%)$. Lymph node form was the most frequent (70.83\%) extrapulmonary form of TB. The only single nucleotide polymorphism detected in the population was c.-392A $>\mathrm{G}$. Genotypes observed were $C Y P 3 A 4 * 1 A / C Y P 3 A 4 * 1 A$ (45.3\%), $C Y P 3 A 4 * 1 A / C Y P 3 A 4 * 1 B(40.6 \%)$ and $C Y P 3 A 4 * 1 B /$ $C Y P 3 A 4 * 1 B(14.2 \%)$, revealing a different distribution with extrapulmonary TB cases $(17.6 \%$ CYP $3 A 4 * 1 A / C Y P 3 A 4 * 1 B$ and $23.5 \% C Y P 3 A 4 * 1 B / C Y P 3 A 4 * 1 B)$. The $C Y P 3 A 4 * 1 A$ allele was found to be associated with tobacco use. The $C Y P 3 A 4^{*} I B$ mutant allele occurred in $34 \%$ of patients. This study revealed that the CYP3A4 5'-upstream regulatory region was highly conserved with the exception of the -392 position. Genotype association with tobacco suggests that CYP3A4 may participate in tobacco metabolism. Genotype distribution inversion
\end{abstract}

Correspondence to: Dr Liane de Castro, Laboratório de Biomarcadores e Hepatotoxicidade, Instituto de Pesquisa Clínica Evandro Chagas - Fundação Oswaldo Cruz (IPEC/FIOCRUZ), Av. Brasil 4365, Manguinhos, Rio de Janeiro 21040-360, RJ, Brazil E-mail: liane.castro@ipec.fiocruz.br

Key words: Brazil, CYP3A4 promoter region, CYP3A4 upstream region sequence analysis, $C Y P 3 A 4^{*} 1 B$, genetic polymorphism, tuberculosis in extrapulmonary TB cases suggests that CYP3A4 may be involved in TB prognosis.

\section{Introduction}

The CYP3A4 enzyme concurs for phase I drug metabolism in humans with specificity for several substrates, and is responsible for the oxidation of many therapeutic drugs (1). Rifampicin, an antibiotic commonly used for tuberculosis (TB) treatment $(2,3)$, protease inhibitors and non-nucleoside reverse transcriptase inhibitors used for human immunodeficiency virus (HIV) therapy $(4,5)$, and many medicines from the anti-inflammatory and antibiotic classes (2-6) have been described as inhibitors or inductors of CYP $3 A$ gene expression $(3,6-8)$.

CYP3A expression is increased by the transcriptional activation intermediated by the pregnane $\mathrm{X}$ receptor (PXR), a nuclear receptor that is the principal activator of $C Y P 3 A$ transcription and which plays a central role in regulating many genes involved in drug metabolism and elimination. In this way, PXR responsive elements on DNA have been considered significant in predicting and preventing drug interactions, metabolism and disposition $(2,9)$.

Considering the involvement of CYP3A4 enzymes in the metabolism of steroids, fatty acids, xenobiotics and the nicotinamide adenine dinucleotide phosphate (NADP)-dependent electron transport pathway $(2,7,10,11)$, we could expect the CYP3A4 metabolic pathway to be directly or indirectly involved in response to infections. Indirect involvement appears to mobilize transcriptional activation factors.

During CYP3A4 transcriptional activation by xenobiotics such as rifampicin, the PXR binds to and is activated by the drug (12). The PXR drug complex forms a heterodimer with the retinoid X receptor (RXR) and binds to the PXR response elements on the CYP3A4 gene promoter (3). Therefore, polymorphisms in this region may interfere with transcription or enzyme activity.

The nuclear vitamin D receptor (VDR) is the receptor with the highest identity sequence to PXR (9). There is evidence for the essential role of VDR in mediating the vitamin D actions. The receptor binds to the hormonal metabolite of vitamin D 1,25-dihydroxyvitamin D3 [1,25(OH) 2 D3]. Similar 
to PXR, the $1,25(\mathrm{OH})_{2} \mathrm{D} 3-\mathrm{VDR}$ complex interacts with RXR, generating a heterodimer. Following interaction, the resultant complex binds to the dihydroxyvitamin $\mathrm{D}(3)$ receptor (DR3) hormone responsive element on DNA (13). It induces calcium/ phosphate translocation in tissues such as bone (resulting in normal bone mineralization and remodeling), intestine and kidney (13). Moreover, there are apparent vitamin D bioactivities in other targets, such as cells of the neural, endocrine and immune systems. In this way, $1,25(\mathrm{OH})_{2} \mathrm{D} 3$ acts as a general suppressor, particularly of T-helper cells (13). Concerning this, VDR and tumor necrosis factor- $\alpha$ (TNF- $\alpha$ ) genes are crucial in the intracellular killing of Mycobacteria tuberculosis (14). In addition, genetic studies have revealed an association between genetic variants of the natural resistance-associated macrophage protein (NRAMP1) and VDR genes and smearpositive pulmonary TB (15).

Evidence regarding the $1,25(\mathrm{OH})_{2} \mathrm{D} 3-\mathrm{VDR}$ complex has shown that after binding to the RXR heterodimer, this complex interacts with the PXR response elements on the CYP3A4 gene promoter $(12,16)$. It suggests that CYP3A4 enzymes or signaling involving CYP3A4 may be associated with susceptibility to the development of pulmonary TB.

There is evidence of CYP3A4 expression in the prostate, breast, colon, small intestine and liver, in which these enzymes amount to $30 \%$ of the total CYP protein content $(8,17-20)$. This may be a result of the significant role of CYP3A4 in the oxidation of testosterone and estrogen, as suggested in studies researching the mechanisms involved in breast cancer $(17,21)$ and androgen-mediated prostate carcinogenesis (16).

The CYP3A4 gene is located on chromosome 7q21.3-q22.1 and consists of 27.592 base pairs and 13 exons. Its 5'-flanking region (5'-untranslated region or UTR) includes a basal transcription element at the -35 to -50 promoter region, an AP-3 binding site, an estrogen response element, a glucocorticoid response element, a hepatocyte nuclear factor-4 (HNF4) element, two HNF-5 elements and a protein p53 binding motif $(22,23)$.

The occurrence of genetic variants of CYP3A4 was noted following the detection of ethnic variation in CYP3A metabolism in comparative studies of triazolam pharmacokinetics and pharmacodynamics between Caucasians and Southern Asians (24). The association with ethnicity was also found in studies analyzing $6 \beta$-hydroxycortisol:cortisol conversion ratios (21).

The first molecular approach in the study of CYP3A4 used conformation sensitive gel electrophoresis (CSGE) of the PCR product to analyze Caucasian males with prostate cancer. The genetic variant $C Y P 3 A 4-V(C Y P 3 A 4 * 1 B)$ was identified, which presented the alteration c. $-392 \mathrm{~A}>\mathrm{G}$ on the $C Y P 3 A 4$ promoter with a frequency $\geq 0.01$. $C Y P 3 A 4 * 1 B$ was associated with a higher tumor lymph node metastasis stage and Gleason grade. The authors concluded that a single base change or single nucleotide polymorphism (SNP) in the 5'-flanking region of the CYP3A4 gene may influence prostate carcinogenesis (16). Following this, the $C Y P 3 A 4 * 1 B$ allele was also detected in other studies analyzing genetic polymorphisms in the 5'-upstream regulatory region and hepatic expression of CYP3A4 (25). Sequencing analysis provided additional evidence of this variant in Caucasian, African-American and Chinese individuals (26).

A posterior haplotype approach supported the association of the $C Y P 3 A 4 * 1 B$ allele with prostate cancer in $C Y P 3 A 4 * 1 B$ and $C Y P 3 A 5^{*} 1$, as well as $C Y P 3 A 4 * 1 B$ and $C Y P 3 A 5^{*} 3$ haplotypes (27).

$C Y P 3 A 4 * 1 B$ has also been associated with the development of breast cancer in females with early-onset menarche from the US (African-American, Hispanic and Caucasian) (28). Conversely, a case-control study found no association between the functional implication of this polymorphism and breast or ovarian cancer in Australian Caucasian women (29).

Regarding the involvement of CYP3A4 in the metabolism of certain TB and HIV treatment drugs, the occurrence of CYP3A4 gene variants, mainly those affecting regulatory regions, interferes in gene transcription, enzyme activity and other regulatory events. Therefore, these variants may influence drug metabolism and cause many adverse effects. Furthermore, polymorphisms in the CYP3A4 5'-upstream region may interfere indirectly in the response to infection, since this region interacts with the VDR-containing complex associated with TB infection. Since CYP3A4 polymorphisms and allelic frequencies in individuals from Rio de Janeiro remain unknown, effort is required to provide knowledge of this issue. In this way, the aim of this study was to research the CYP3A4 5'-upstream region in Brazilian TB patients co-infected or not with HIV, and to investigate whether genotypes could be involved in the evolution of the TB infection.

\section{Materials and methods}

Subjects, settings and data collection. This study was performed at the Laboratório de Biomarcadores e Hepatotoxicidade, Instituto de Pesquisa Clínica Evandro Chagas, FIOCRUZ (IPEC-FIOCRUZ), Rio de Janeiro, Brazil. The IPECFIOCRUZ Ethics Committee approved this research under the SISNEP register: 0013.0.009.000-03/2003. Enrollment was carried out at the IPEC-FIOCRUZ Hospital, a reference center for infectious diseases, including TB and HIV, providing both in- and out-patient treatment. Blood samples and clinical information were obtained from 106 patients undergoing TB treatment, with or without HIV coinfection. In this follow-up study, all patients eligible were invited to participate. Patients were randomly selected in the period from October 2004 to October 2009. Eligibility criteria were i) a sputum smear with acid-fast bacilli or culture positive for Mycobacterium tuberculosis or documented diagnosis elsewhere; ii) signed written consent and iii) HIV serology. Exclusion criteria were i) age $<18$ years and ii) lack of 5'-upstream CYP3A4 genotyping results.

The main variables were gender, HIV status, tobacco use, skin color classification and TB clinical form. HIV status was defined as negative or positive depending on the serology result in the patient's medical chart. Tobacco use was classified as positive (smoker) when patients answered yes to the questionnaire about smoking in the medical records. The diagnosis of TB was based on the recommendations of the Brazilian Ministry of Health described in the National Program for Tuberculosis Control (30).

TB clinical presentation (clinical form) was defined as pulmonary, extrapulmonary or disseminated form following the information in the patient's medical chart. Pulmonary $\mathrm{TB}$ was diagnosed through medical evaluation for TB in the lungs, which included medical history, physical examination, 
Table I. Population characteristic distribution in relation to gender.

\begin{tabular}{lcccc}
\hline & Female $(\mathrm{n}=34 ; \%)$ & Male $(\mathrm{n}=72 ; \%)$ & Total $(\mathrm{n}=106 ; \%)$ & Statistical test \\
\hline $\begin{array}{l}\text { Skin color } \\
\text { White }\end{array}$ & $16(47.06)$ & $33(45.83)$ & $49(46.23)$ & Chi-square $(1 \mathrm{df})=0.008$ \\
Non-white & $18(52.94)$ & $39(54.17)$ & $57(53.77)$ & 0.9278 \\
HIV & & & & Chi-square $(1 \mathrm{df})=5.329$ \\
Negative & $24(70.59)$ & $32(44.44)$ & $56(52.83)$ & 0.021 \\
Positive & $10(29.41)$ & $40(55.56)$ & $50(47.17)$ & \\
\hline
\end{tabular}

chest X-ray and microbiological examination (by identifying the Mycobacterium tuberculosis in a sputum sample). Extrapulmonary TB was diagnosed in patients with TB affecting areas other than the lungs. To obtain information on infection evolution, the subcategories of extrapulmonary forms were analyzed. These cases were described depending on the location of affected organs and/or tissue. Disseminated TB was diagnosed as a TB infection that had spread from the lungs to other parts of the body through the blood or lymph system.

DNA sample and extraction. Peripheral blood samples $(5 \mathrm{ml})$ collected from each patient were stored at $-20^{\circ} \mathrm{C}$ in sterile EDTA vacutainer tubes until DNA extraction. Genomic DNA extraction was performed using the QIAamp DNA Blood Mini kit (Qiagen, Santa Clara, CA, USA) following the manufacturer's instructions.

CYP3A4 5'-upstream amplification. CYP3A4 5'-upstream region amplification was obtained by polymerase chain reaction (PCR) resulting in a 661-bp product, using primers designed based on the Ensembl Gene Report for ENSG00000160868 (CYP3A4 gene, CYP3A4-202 transcript, exon info ENST00000336411) (31) with the NCBI primer tool (32). The forward primer was $C Y P 3 A 4 F$ (5'-TGAGGAGCTCACCTCTGTTC-3') and the reverse primer was $C Y P 3 A 4 R$ (5'-GAGCAACACAGAGCTGAAAGG-3'); these were designed for annealing specifically at position -698 to $-679(C Y P 3 A 4 F)$ and -58 to $-38(C Y P 3 A 4 R)$ in the $C Y P 3 A 4$ gene.PCR was performed in a $50-\mu 1$ mixture containing genomic DNA, 2.5 units of AmpliTaq DNA polymerase (Invitrogen), $20 \mathrm{pmol}$ of each primer, $0.2 \mathrm{mM}$ of each dNTP (Invitrogen), $1.5 \mathrm{mM} \mathrm{MgCl}_{2}$ and $1 \mathrm{X}$ PCR buffer (Invitrogen). Following initial denaturation at $95^{\circ} \mathrm{C}$ for $5 \mathrm{~min}, 35$ cycles of $94^{\circ} \mathrm{C}$ for $1 \mathrm{~min}, 59.5^{\circ} \mathrm{C}$ for $1 \mathrm{~min}$ and $72^{\circ} \mathrm{C}$ for $2 \mathrm{~min}$ were performed. Next, a final elongation at $72^{\circ} \mathrm{C}$ for $10 \mathrm{~min}$ and a hold cycle at $4^{\circ} \mathrm{C}$ were performed. Following gel electrophoresis of a small quantity of each sample to confirm amplification, PCR product purification using the Wizard kit (Promega) was performed, following the quantitative analysis by gel electrophoresis using low mass DNA ladder and DNA sequencing using 1 pmol of each primer. $C Y P 3 A 4$ 5'-upstream genotype analysis was performed by analysis of electropherograms using sequences obtained with reverse and forward primers, simultaneously, for each sample. The reaction products were analyzed on the ABI Prism ${ }^{\circledR} 3730$ DNA Analyser at 'Plataforma GenômicaSequenciamento de DNA-RPT01A-PDTIS/FIOCRUZ'.
CYP3A4 5'-upstream genotype determination. The description of sequence variations was based on GenBank sequence AF280107 of the CYP3A4 gene (32) and the Ensembl Gene Report for ENSG00000160868 (CYP3A4 gene, complete sequence $C Y P 3 A 4-001$ transcript, exon info transcript ID ENST00000336411; 13 exons; transcript length 2.153 bp; translation length 503 residues) (31). The sequences were edited and assigned using Sequencer 4.1.4 software. The GenBank sequence AF280107 (32) was used as a reference for comparisons of DNA using Sequencer 4.1.4. The region analyzed was -698 to -38 of the CYP3A4 gene, which corresponds to positions 61340 to 62000 in AF28017 Nucleotide Loccus in GenBank and 99382402 to 99381742 chromosome bp - system used to register variations in Ensembl (Line Numbering Relative to Coordinate Systems).

Positions of nucleotides are given as distances in base pairs from the first ATG (methionine translational start signal), in which $\mathrm{A}$ is the nucleotide +1 and its adjacent 3 ' nucleotide is -1. The A base of $C Y P 3 A 4$ 's first ATG is found at position number 62037 in the GenBank entry identified by accession number AF280107. Guidelines for naming variants were NCBI and Ensembl patterns, which follow the recommendations of the human nomenclature committee (33). The search for variants in the 5'-upstream regulatory region of $C Y P 3 A 4$ included positions previously identified with DNA sequence variants described previously, revised by Keshava et al (34) and available in the Ensembl SNP register of December 6, 2009.

To allow greater convenience and speed in the comparative analysis of the sequences using Sequencer, the positions of variants in the amplicon (position in amplicon) were determined considering the first base of the forward primer ( 5 ' end) as base 1 and the last base of the forward primer as base 661 . After that, in results, the conventional position based on GenBank and Ensembl was shown.

Statistical analysis. Proportions (\%) for the categorical data of gender, skin color, smoking, TB clinical form, TB extrapulmonary forms, CYP3A4 alleles and genotype distribution were used to describe characteristics in the studied population. Data analysis involved comparisons of proportions and analysis of the association of genotypes with the clinical characteristics. The Pearson Chi-square or Fisher's exact tests were used for categorical variables in the contingency tables analysis. A p-value of $\mathrm{p}<0.05$ or $5 \%$ was considered significant. All analyses were carried out using the R software (package Epicalc). 
Table II. Tuberculosis (TB) clinical forms diagnosed.

No. $\%$

\begin{tabular}{lrr}
\hline TB clinical form & & \\
Disseminated & 29 & 27.36 \\
Extrapulmonary & 24 & 22.64 \\
Pulmonary & 51 & 48.11 \\
Missing & 2 & 1.88 \\
Extrapulmonary TB & & \\
Skin & 2 & 8.33 \\
Lymph nodes & 17 & 70.83 \\
Larynx & 1 & 4.17 \\
Ophthalmic & 3 & 12.50 \\
Oropharyngeal & 1 & 4.17 \\
\hline
\end{tabular}

\section{Results}

Patient characteristics. The demographic and clinical characteristics of the patients concerning gender are described in Table I. The majority of patients were male (67.9\%), who showed the highest frequency of HIV infection $(47.2 \%$, $\mathrm{p}=0.021)$. Regarding patient skin color, the majority $(53.8 \%)$ was classified as non-white (black or brown skin color). The skin color distribution was homogenous with relation to gender (Table I).

As shown in Table II, TB clinical forms observed were pulmonary $(48.1 \%)$, extrapulmonary $(22.6 \%)$ and disseminated (27.3\%). Among extrapulmonary cases, the majority was lymph node form (70.83\%) and other forms were much less frequent (Table II).

Regarding tobacco use, it was observed that $30.2 \%$ of patients were tobacco users against $65.1 \%$ that were not (Table III). Tobacco use was more frequent in males $(81.25 \%$ vs. $18.75 \%$ in females), but without significant association. Considering TB clinical form, tobacco use was more frequent in pulmonary TB cases, followed by disseminated and extrapulmonary forms, respectively (Table III).

CYP3A4 sequence analysis. The primers designed allowed coverage of the entire regulatory region and upstream region and beyond that, permitting the description of nearly $600 \mathrm{bp}$ for each patient. Comparative analysis of the DNA sequence of patients and comparisons between them and the reference sequence (GenBank AF280107) revealed that the analyzed region in all 106 studied individuals was very similar to the reference sequence. Performing a direct search focusing on the 20 SNP regions registered in the Ensembl genomic database, it was observed that 19 of the 20 analyzed positions revealed the same bases corresponding to the wild reference sequence.

The genotypes identified in the patients are shown in Table IV, which shows the bases in Brazilian samples corresponding to each one of the SNPs described in the Ensembl database. By searching the literature and the Ensembl SNP databank, it was verified that to date 20 positions have been identified with SNP in the CYP3A4 studied region. The percentage of homozygosity was $100 \%$ for almost all positions with the exception of position c.-392, which carried CYP3A4*IA alleles (characterized for the 'a' base) or CYP3A4*IB (characterized for the ' $\mathrm{g}$ ' base). The distribution of genotypes at this polymorphic region is shown in Table IV, and the classification of alleles and genotypes is shown in Table V. The percentage of homozygosity at position c.-392 was 59.5\% (48 AA + 15 GG in a total of 106) and the percentage of heterozygosity was $40.5 \%$ (43 AG). The haplotype count was 139 to A [(48 AA x 2) + $43 \mathrm{AG})]$ and 73 to $\mathrm{G}[(15 \mathrm{GG} \times 2)+(43 \mathrm{AG})]$. The sum of haplotypes was 212 (139 AA + 73 GG). Then, considering 212 as the $\mathrm{n}$ value or $100 \%$, it was observed that $\mathrm{A}$ haplotypes $(C Y P 3 A 4 * 1 A)$ were equivalent to $66.0 \%$ (variant frequency of 0.66), while $\mathrm{G}$ haplotypes $\left(C Y P 3 A 4^{*} I B\right)$ corresponded to $34.0 \%$ (variant frequency of 0.34 ).

CYP3A4 and clinical characteristics. The distribution of CYP3A4 genotypes considering clinical characteristics is shown in Table VI. A significant correlation was found between CYP $3 A 4 * 1 A$ and tobacco consumption $(\mathrm{p}=0.0145)$. The $13.3 \%$ of consumers registered for $\mathrm{GG}$ patients $\left(C Y P 3 A 4^{*} 1 B\right)$ increased to $25.6 \%$ in individuals carrying only one copy of the $C Y P 3 A 4 * 1 A$ (AG) allele and reached $39.6 \%$ among the AA homozygous individuals. With regard to TB clinical form, the disseminated form AG and AA genotypes revealed similar frequencies (44.8 and $41.4 \%$, respectively), while the GG genotype was rare (13.8\%). Among TB extrapulmonary forms, AA genotype was the most frequent (54.2\%), followed by AG (29.2\%) and GG (16.7\%) genotypes. Among TB pulmonary form, AG and AA genotypes were the most frequent ( 45.1 and $41.2 \%$, respectively), while the GG genotype was less frequent (13.7\%). The same was observed among TB extrapulmonary forms, in which lymph node forms were the most frequent (70.8\%); the AA genotype being the most frequent (58.8\%), followed by the GG and AG genotypes identified in 23.5 and $17.6 \%$ of patients, respectively. With regard to HIV status and skin color, a similar distribution of the various genotypes was observed.

\section{Discussion}

Patient characteristics. The high percentage of TB-HIV-positive serology among patients was a predictable result due to the fact that IPEC is a reference center of HIV and TB-HIV. Additionally, the frequency of positive serology for HIV being significantly higher among males $(\mathrm{p}=0.021)$ is in correlation with the HIV prevalence in males registered in Brazil: 22 per 100,000 against 13.9 per 100,000 in females in 2007 (35).

Regarding the differential allelic expression of CYP3A4 associated with many xenobiotics (36), and the previous evidence indicating that smoking is causatively associated with active TB $(37,38)$, alterations in the CYP3A4 regulatory region may influence the activity or expression of the enzyme, and could be related to variation in drug interaction, including tobacco compounds. In this study, the tobacco consumption frequency among patients was twice (32\%) as high as that previously described in the Brazilian population; approximately $16 \%$, ranging from $9.5 \%$ in Salvador to $21.2 \%$ in Porto Alegre and Rio Branco cities (39). Additionally, when each TB clinical form was analyzed separately, $15.62 \%$ of the patients with the extrapulmonary form of TB were smokers. This number increased to $25 \%$ among disseminated cases and to 
Table III. Consumption of tobacco in patients considering gender and tuberculosis (TB).

\begin{tabular}{|c|c|c|c|c|}
\hline & $\begin{array}{c}\text { No tobacco consumed } \\
\text { No. }(\%)\end{array}$ & $\begin{array}{c}\text { Tobacco consumed } \\
\text { No. }(\%)\end{array}$ & $\begin{array}{c}\text { Total } \\
\text { No. }(\%)\end{array}$ & p-value \\
\hline $\begin{array}{l}\text { Gender } \\
\text { Female } \\
\text { Male }\end{array}$ & $\begin{array}{l}26(37.68) \\
43(62.32)\end{array}$ & $\begin{array}{r}6(18.75) \\
26(81.25)\end{array}$ & $\begin{array}{l}32(31.68) \\
69(68.32)\end{array}$ & $\begin{array}{l}\text { Chi-square test } 0.0944 \\
\quad(1 \mathrm{df})=2.798\end{array}$ \\
\hline $\begin{array}{l}\text { TB clinical form } \\
\text { Disseminated } \\
\text { Extrapulmonary } \\
\text { Pulmonary }\end{array}$ & $\begin{array}{l}18(26.09) \\
19(27.54) \\
31(44.93)\end{array}$ & $\begin{array}{r}8(25.00) \\
5(15.62) \\
19(59.38)\end{array}$ & $\begin{array}{l}26(25.74) \\
24(23.76) \\
50(49.50)\end{array}$ & Fisher's exact test 0.4618 \\
\hline
\end{tabular}

Table IV. CYP3A4 upstream region analysis in Brazilian patients and the bases to each region previously described with SNPs in the literature.

\begin{tabular}{|c|c|c|c|}
\hline $\begin{array}{l}\text { Distance from } \\
\text { first ATG }\end{array}$ & $\begin{array}{c}\text { Position in } \\
\text { GenBank (AF280107) }\end{array}$ & $\begin{array}{l}\text { Mutation (chromosome } \\
\text { bp in Ensembl) }\end{array}$ & $\begin{array}{c}\text { Genotype detected } \\
(\%)\end{array}$ \\
\hline-655 & 61382 & $99382359^{a}$ & AA (100) \\
\hline-630 & 61407 & 99382334:A/G & AA (100) \\
\hline-605 & 61433 & 99382309:A/G & AA (100) \\
\hline-529 & 61509 & 99382233:T/C & TT (100) \\
\hline-486 & 61551 & 99382190:G/A & GG (100) \\
\hline-444 & 61593 & 99382148:T/G & TT (100) \\
\hline-402 & 61635 & 99382106:G/A & GG (100) \\
\hline-392 & 61645 & 99382096:G/A & AA (45.3); AG (40.6); GG (14.2) \\
\hline-386 & 61651 & 99382090:A/G & AA (100) \\
\hline-369 & 61668 & 99382073:T/A & TT (100) \\
\hline-320 & 61717 & 99382024:G/A & GG (100) \\
\hline-301 & 61736 & 99382005:T/C & TT (100) \\
\hline-290 & 61749 & 99381994:A/G & $\mathrm{AA}(100)$ \\
\hline-246 & 61790 & 99381951:-/GT & ---- (100) \\
\hline-219 & 61818 & 99381923:A/C & AA (100) \\
\hline-179 & 61858 & 99381883:T/C & TT (100) \\
\hline-156 & 61881 & 99381860:C/A & CC (100) \\
\hline-120 & 61917 & 99381824:A/C/G & AA (100) \\
\hline-66 & 61971 & $99381770^{\mathrm{a}}$ & CC (100) \\
\hline-62 & 61975 & 99381766:C/A & CC (100) \\
\hline
\end{tabular}

${ }^{\text {a } V a r i a n t s ~ d e s c r i b e d ~ i n ~ t h e ~ l i t e r a t u r e, ~ b u t ~ n o t ~ r e g i s t e r e d ~ i n ~ t h e ~ E n s e m b l ~ S N P ~ s e c t i o n . ~}$

Table V. Variants at position c.-392 in the CYP3A4 upstream region in Brazilian patients.

\begin{tabular}{lccr}
\hline Alelles & Classification & Genotype (bases) & No. \\
\hline$C Y P 3 A 4 * 1 A$ & Wild-type & AA & 48 \\
$C Y P 3 A 4^{*} 1 B$ & Mutant & AG & 45.3 \\
$C Y P 3 A 41 A+C Y P 3 A 4 * 1 B$ & Heterozygote & GG & 14.2 \\
\hline
\end{tabular}

$59.4 \%$ among patients with pulmonary TB. This indicates that among pulmonary TB patients, the percentage of smokers was 3.7 times higher than that observed in the Brazilian population. In this way, besides the absence of a significant association between smoking and TB clinical form or extrapulmonary TB, it was verified that smokers were the majority among patients with pulmonary TB. These results suggest that smoking may be related to pulmonary $\mathrm{TB}$, which correlates with previous 
Table VI. Genotype distribution and clinical characteristics observed in Brazilian patients.

\begin{tabular}{|c|c|c|c|c|c|}
\hline & AA & AG & GG & Total & p-value \\
\hline Gender & & & & & 0.9849 \\
\hline Female & $15(31.25)$ & $14(32.56)$ & $5(33.33)$ & $34(32.08)$ & Chi-square $(2 \mathrm{df})=0.031$ \\
\hline Male & $33(68.75)$ & $29(67.44)$ & $10(66.67)$ & $72(67.92)$ & \\
\hline Total & $48(45.28)$ & $43(40.57)$ & $15(14.15)$ & & \\
\hline Skin color & & & & & 0.4195 \\
\hline White & $25(52.08)$ & $19(44.19)$ & $5(33.33)$ & $49(46.23)$ & Chi-square $(2 \mathrm{df})=1.738$ \\
\hline Non-white & $23(47.92)$ & $24(55.81)$ & $10(66.67)$ & $57(53.77)$ & \\
\hline HIV & & & & & 0.5546 \\
\hline Negative & $26(54.17)$ & $24(55.81)$ & $6(40.00)$ & $56(52.83)$ & Chi-square $(2 \mathrm{df})=1.179$ \\
\hline Positive & $22(45.83)$ & $19(44.19)$ & $9(60.00)$ & $50(47.17)$ & \\
\hline \multicolumn{6}{|l|}{ Tobacco use } \\
\hline No & $24(50.00)$ & $32(74.42)$ & $13(86.67)$ & $69(65.09)$ & 0.0145 \\
\hline Missing & $5(10.42)$ & $0 \quad(0.00)$ & $0 \quad(0.00)$ & $5(4.72)$ & Fisher's exact \\
\hline Yes & $19(39.58)$ & $11(25.58)$ & $2(13.33)$ & $32(30.19)$ & \\
\hline \multicolumn{5}{|l|}{ TB clinical form } & 0.8411 \\
\hline Disseminated & $12(25.00)$ & $13(30.23)$ & $4(26.67)$ & $29(27.36)$ & Fisher's exact \\
\hline Extrapulmonary & $13(27.08)$ & $7(16.28)$ & $4(26.67)$ & $24(22.64)$ & \\
\hline Pulmonary & $21(43.75)$ & $23(53.49)$ & $7(46.67)$ & $51(48.11)$ & \\
\hline Chemoprop & $1(2.08)$ & $0 \quad(0.00)$ & $0 \quad(0.00)$ & $1(0.94)$ & \\
\hline Missing & $1 \quad(2.08)$ & $\begin{array}{ll}0 & (0.00)\end{array}$ & $\begin{array}{ll}0 & (0.00)\end{array}$ & $1 \quad(0.94)$ & \\
\hline \multicolumn{5}{|l|}{ Extrapulmonary TB } & 0.5737 \\
\hline Skin & $1 \quad(7.69)$ & $1(14.29)$ & $0 \quad(0.00)$ & $2(8.33)$ & Fisher's exact \\
\hline Lymph nodes & $10(76.92)$ & $3(42.86)$ & $4(100.00)$ & $17(70.83)$ & \\
\hline Larynx & $0 \quad(0.00)$ & $1(14.29)$ & $0 \quad(0.00)$ & 1 (4.17) & \\
\hline Ophtalmic & $1(7.69)$ & $2(28.57)$ & $0 \quad(0.00)$ & $3(12.5)$ & \\
\hline Oropharyngeal & $1(7.69)$ & $0 \quad(0.00)$ & $\begin{array}{ll}0 & (0.00)\end{array}$ & 1 (4.17) & \\
\hline
\end{tabular}

data linking smoking and active TB $(37,38)$. Additional studies using a case-control approach may clarify this issue.

CYP3A4 sequence analysis. The screening of SNPs in the CYP3A4 upstream region in Brazilian patients based on a Human Genome Epidemiology Review (34) and the Ensembl databank revealed that almost all SNPs were absent in the studied population. These SNPs were identified in Asian individuals originating from Japanese and Chinese populations (34). In this study, the SNP c.-392A>G was the only sequence variation identified in Brazilian patients undergoing TB treatment at the IPEC Hospital in Rio de Janeiro. This SNP (c.-392A $>\mathrm{G}$ ) corresponds to $C Y P 3 A 4-V$ previously detected in a study of Caucasian volunteers by Rebbeck et al (16). Westlind et al obtained similar results when analyzing the most proximal 5'-upstream region from +10 to c. $-490 \mathrm{bp}$, which contains the CYP $3 A 4$ promoter, in 39 individuals, through the sequencing of nested PCR amplicons. It was observed that the only variation was the $A>G$ mutation at position c. -290 corresponding to CYP3A4-V (25). Our results support those of previous studies indicating that this CYP3A4 gene is highly conserved, and concur with previous data demonstrating that the upstream region is highly preserved $(25,40)$. Moreover, the variation of CYP3A4 among populations reinforces the necessity of studying this gene in Brazilian individuals.
Also termed $C Y P 3 A 4 * 1 B, C Y P 3 A 4-V$ represents the most common polymorphism observed in the promoter region in the nifedipine-specific response element (NFSE) with a change at position c.-392 from A to $\mathrm{G}$ (agggcaagag to agggcaggag) (16). However, this variant is presented as a $\mathrm{G}$ to A mutation in the Ensembl databank (99382096:G/A).

CYP3A4 and clinical characteristics. CYP3A4-V frequency was approached mainly in prostate cancer and leukemia studies $(16,40,41)$. Initially, researchers focused on searching for an association between genotypes and cancer development. Then, a novel approach was described, searching for the association of genotypes with cancer prognosis, reflected by a correlation between $C Y P 3 A 4-V$ and the high aggressiveness of prostate cancer (42).

In this study, no association was found between a specific CYP3A4 genotype and the development of a particular TB clinical form. However, in the pulmonary and disseminated forms of TB, it was observed that the AG genotype (wild-type or $C Y P 3 A 4-V$ ) was slightly more frequent than the AA genotype. Furthermore, among TB extrapulmonary forms, the AA genotype (wild-type) was the most frequent, particularly when focusing on the most commonly observed lymph node forms. These results suggest that there is a possibility of finding an association between the CYP3A4 genotypes in the upstream 
regulatory region and TB clinical form. Case-control studies comparing genotype distribution between TB and non-TB patients may provide useful information to clarify this.

The majority of clinical studies involving $C Y P 3 A 4$ have used the frequency of $C Y P 3 A 4-V$ as a tool to analyze associations with cancers that are more common in a specific ethnic group. An investigation comparing ethnicities performed by Walker et al detected that $C Y P 3 A 4-V$ was more frequent in African Americans, being observed in $53 \%$ of cases. In Caucasians, it was presented in only $9 \%$, whereas in Taiwanese it was not observed ( $0 \%)$. Conversely, the wild-type allele $(C Y P 3 A 4 * 1 A$, agggcaagag) was the most frequent form in Caucasians and the Taiwanese (41). Zeigler-Johnson et al found similar results in studies focusing on prostate carcinogenesis. Analyzing genotypes at the SRD5A2 and $C Y P 3 A 4$ loci, the authors detected the highest frequency of $C Y P 3 A 4-V$ in Ghanaians and African Americans ( $>50 \%$ ), while in Caucasians it was less than $10 \%$ and in Asians it was non-existent (40).

$C Y P 3 A 4 * 1 B$ (as $C Y P 3 A 4-V$ ) was previously studied in Brazilian patients by Fiegenbaum et al investigating the role of $A B C B 1, C Y P 3 A 4$ and $C Y P 3 A 5$ genes in simvastatin treatment in patients living in Rio Grande do Sul (43). The same group performed a cross-sectional study investigating possible associations between polymorphisms in genes related to estrogen biosynthesis and estrogen catabolism. In both studies, SNP $C Y P 3 A 4-V$ was the only $C Y P 3 A 4$ variant searched for in the upstream region (44). Since these investigations used PCR and restriction mapping analysis as a molecular biology approach, these punctual studies assumed $C Y P 3 A 4-V$ to be the only possible variation without a previous SNP screening.

In this regard, the present study provides additional data, providing the largest vision of the $C Y P 3 A 4$ upstream regulatory region, obtained through the first attempt at sequencing performed on Brazilian individuals. Moreover, our results indicate the possibility of using direct $C Y P 3 A 4-V$ focus in Brazilian individuals as PCR and RFLP, although other studies with populations originating in other Brazilian states and with different ethnicity are required for a secure analysis. Furthermore, it is also necessary to remember that these previous studies of CYP3A4 in Brazilian individuals have analyzed those of European descent, while this study focused on individuals living in a southeastern state with different ethnicity. Brazil presents a large miscegenation of native American Indians, European Caucasians and African blacks that originate from various countries. Due to Brazil's large size, topographic diversity and colonization history, different regions show singular prevalence of ancestry with reflects on the population subtypes (45). This justifies the need for studies focusing on different regions of Brazil and different population subtypes.

The haplotype analysis in this study revealed that $C Y P 3 A 4 * 1 B$ was observed in $34 \%$ of patients and $C Y P 3 A 4 * 1 A$ in $66 \%$. Thus, the $C Y P 3 A 4 * 1 B$ frequency was much lower than that observed in African Americans $(40,41)$ and Ghanaians (40), while CYP3A4*1A was the highest, although lower than the $90-100 \%$ described in Caucasians and Asians, respectively (40). These results demonstrate that, in Brazilians, $C Y P 3 A 4 * 1 B$ and $C Y P 3 A 4 * 1 A$ have a peculiar distribution. Although many studies have connected $C Y P 3 A 4$ genotypes with ethnicity $(36,40,41,46-48)$, in this study the genotype distribution revealed no correlation with patient skin color. Besides this, it was observed that the GG genotype was twice as common in non-white people (66.7\%). Regarding the AG genotype, most carriers were non-white, while with the AA genotype most were white.

Besides the miscegenation in the population analyzed in this study, which causes difficulty when making comparisons, a similar tendency was also observed in the haplotype distribution with relation to skin color in investigations performed by Schirmer et al. They observed that $C Y P 3 A 4 * 1 A$ exhibited a high haplotype homozygosity (AA haplotypes) in European Caucasians, while it was low in African Americans. In other words, individuals of African descent demonstrated a high level of homozygosity to $C Y P 3 A 4 * 1 B$ (GG haplotypes) and AG heterozygous haplotypes. Since CYP3A4 is involved in vitamin D metabolism and carriers of $C Y P 3 A 4 * 1 B$ allelles exhibit higher expression of this enzyme, it is hypothesized that the deficiency in Vitamin D due to decreased ultraviolet intensity could be a selecting factor responsible for the apparent elimination of $C Y P 3 A 4 * 1 B$ alleles, in non-African populations (46).

Considering that the active form of vitamin $D$ $\left(1,25(\mathrm{OH})_{2} \mathrm{D} 3\right)$ has been shown by in vitro studies to inhibit the growth of Mycobacterium tuberculosis through activating monocytes and cell-mediated immunity, it was suggested that vitamin D status may be associated with the risk of TB development (49). It was reinforced in a recent case-control study involving 166 Vietnamese TB patients confirming the association between vitamin D deficiency and TB in man $(p=0.01)$ (50). Previous investigations have found that 25-hydroxycholecalciferol (serum vitamin D) deficiency or absence was associated with active TB and an association was detected between the combination of VDR genotypes and 25-hydroxycholecalciferol deficiencies with TB development (51). In this study, involving $45.3 \%$ of patients carrying AA and $40.6 \%$ of patients carrying AG genotypes, a total of $85.9 \%$, it would be expected that most individuals demonstrated a functional vitamin $\mathrm{D}$ metabolism and a satisfactory immune response to TB. In this way, other clinical and genetic studies concerning VDR and the CYP3A4 upstream region together may clarify the possibility of using these genetic regions for TB prognosis and prediction.

Clinical research (47) and in vitro functional analysis $(25,48)$ generated controversial results concerning the affects of carrying $C Y P 3 A 4-V$. Studies of patients with the wild-type genotype $\left(C Y P 3 A^{*} 1 A\right.$ or $\left.C Y P 3 A 4-W\right)$ found that these individuals may be at an increased risk of treatmentrelated leukemia. The authors suggested that it may be a result of epipodophyllotoxin and other chemotherapeutic agent metabolism by $C Y P 3 A 4$. In this way, $C Y P 3 A 4-V$ may damage the metabolism of anticancer drugs, reducing the production of potentially DNA-damaging reactive intermediates (47). Beyond the suggested roles in cancer response and TB immune response, the CYP3A4 gene may be explored in approaches for the prediction of therapy response in AIDS and TB, since CYP3A4 demonstrates a role in the metabolism of anti-HIV and anti-TB drugs (2-8).

Besides analysis using a human liver microsome system, which found no apparent correlation between CYP3A4 genotypes and nifedipine oxidation activity (25), and in vitro tests using a liver, which revealed that $C Y P 3 A 4 * 1 B$ has only a 
moderate effect on mRNA and protein construction (46), there is evidence linking the $C Y P 3 A 4$ polymorphism and $C Y P 3 A 4$ mRNA production and protein expression through clinical approaches. Transcriptional and genotyping analysis using liver samples from 18 Caucasian donors found an association between allelic constitution and CYP3A4 mRNA levels. Due to this, the authors suggested that $C Y P 3 A 4$ alleles or haplotypes lead to the total hepatic $C Y P 3 A 4 \mathrm{mRNA}$, producing a different enzymatic level that may have an effect on the metabolic capability, as indirectly measured through testosterone 6 $\beta$-hydroxylation (36).

Therefore, comparative analysis combining clinical and social characteristics of TB patients with CYP3A4 genotypes may provide crucial information on the possibility of involvement of the CYP3A4 polymorphism in disease prognosis and drug response in patients. Furthermore, considering the association between VDR variants and pulmonary TB infection previously described (14), given that VDR-containing complex binds to the $C Y P 3 A 4$ 5'-upstream region, it may be expected that a particular polymorphism in the $C Y P 3 A 4$ regulatory region would be associated with pulmonary TB.

In this study, a differential distribution between genotypes and TB infection was found focusing on the extrapulmonary TB forms. The lymph node cases were the most frequent (70.83\%), of which $58.8 \%$ were diagnosed in individuals with the AA genotype. This result concurs with the highest AA frequencies, but the percentage of the other genotypes shows an inverse distribution; GG genotype (23.5\%) being more frequent than AG (17.6\%). Besides the absence of association, in these cases the inversion in genotype distribution reinforces the possibility that $C Y P 3 A 4$ could be involved in TB prognosis. Regarding extrapulmonary TB, cases of absence of the rarest forms among genotypes impaired carry out association tests, as observed in the larynx TB form in AA patients, oropharyngeal TB for AG patients and skin, laryngeal, oropharyngeal and ophthalmic TB for GG genotypes. In this way, investigations over longer periods with an augmented population and case-control studies involving non-TB patients would provide additional information on the participation of CYP3A4 in response to the TB infection. The possibility of $C Y P 3 A 4$ involvement in patient response to diseases has been previously described with regard to cancer prognosis in many regions around the world $(16,17,21,28,47)$, and more recently for TB infection (14).

To conclude, this study represents the first attempt made to analyze the $C Y P 3 A 4$ upstream region by sequencing in Brazilians with mixed-race ancestry. The similarity of CYP3A4 among patients and also the comparison of CYP3A4 Brazilian characteristics to the reference sequence in GeneBank concur with previous reports indicating that the upstream region of this gene is highly conserved. Furthermore, the association between $C Y P 3 A 4$ genotypes and tobacco use suggests that CYP3A4 enzymes may be involved in tobacco compound metabolism. Considering that tobacco is involved in TB susceptibility, it reinforces the necessity of additional studies aiming to understand this association in a physiological and clinical context. Moreover, further longitudinal investigations monitoring extrapulmonary TB could help to clarify the involvement of $C Y P 3 A 4$ genotypes in TB prognosis.

\section{Acknowledgements}

The authors are grateful to the Director of the Evandro Chagas Clinical Research Institute (IPEC), Dr Valdilea Veloso, for providing the facilities to conduct the study. They also thank Dr Marli J. Martins for the helpful discussion, Dr Sergio Pereira Monteiro for the technical assistance, and Leonardo Rosadas and Patricia Silva for the technical support. The authors sincerely thank all patients for their participation in this study. They also thank the Genomic Platform DNA Sequencing (PDTIS-FIOCRUZ). The Foundation for Research Support of Rio de Janeiro State (FAPERJ) and the Programa Estratégico de Apoio À Pesquisa Em Saúde (PAPES-FIOCRUZ) provided financial support.

\section{References}

1. Waxman DJ, Attisano C, Guengerich FP and Lapenson DP: Human liver microsomal steroid metabolism: identification of the major microsomal steroid hormone 6 beta-hydroxylase cytochrome P-450 enzyme. Arch Biochem Biophys 263: 424-436, 1988.

2. Ma X, Cheung C, Krausz KW, et al: A double transgenic mouse model expressing human pregnane $\mathrm{X}$ receptor and cytochrome P450 3A4. Drug Metab Dispos 36: 2506-2512, 2008.

3. Goodwin B, Hodgson E and Liddle C: The orphan human pregnane $\mathrm{X}$ receptor mediates the transcriptional activation of CYP3A4 by rifampicin through a distal enhancer module. Mol Pharmacol 56: 1329-1339, 1999.

4. Mugundu GM, Hariparsad N and Desai PB: Impact of ritonavir, atazanavir and their combination on the CYP3A4 induction potential of efavirenz in primary human hepatocytes. Drug Metab Lett 4: 45-50, 2010.

5. Von Hentig N and Lotsch J: Cytochrome P450 3A inhibition by atazanavir and ritonavir, but not demography or drug formulation, influences saquinavir population pharmacokinetics in human immunodeficiency virus type 1 -infected adults. Antimicrob Agents Chemother 53: 3524-3527, 2009.

6. Flockhart D: Cytochrome P450 Drug Interaction Table. Indiana University School of Medicine, 2007.

7. Rendic S and di Carlo FJ: Human cytochrome P450 enzymes: a status report summarizing their reactions, substrates, inducers, and inhibitors. Drug Metab Rev 29: 413-580, 1997.

8. Guengerich FP: Cytochrome P-450 3A4: regulation and role in drug metabolism. Ann Rev Pharmacol Toxicol 39: 1-17, 1999.

9. Kliewer SA: Pregnane X receptor: predicting and preventing drug interactions. Thrombosis Res 117: 133-136; discussion 145-151, 2005.

10. Niwa T, Yabusaki Y, Honma K, et al: Contribution of human hepatic cytochrome P450 isoforms to regioselective hydroxylation of steroid hormones. Xenobiotica 28: 539-547, 1998.

11. Shou M, Korzekwa KR, Brooks EN, Krausz KW, Gonzalez FJ and Gelboin HV: Role of human hepatic cytochrome P450 1A2 and $3 \mathrm{~A} 4$ in the metabolic activation of estrone. Carcinogenesis 18: 207-214, 1997.

12. Thompson PD, Jurutka PW, Whitfield GK, et al: Liganded VDR induces CYP3A4 in small intestinal and colon cancer cells via DR3 and ER6 vitamin D responsive elements. Biochem Biophys Res Commun 299: 730-738, 2002.

13. Jurutka PW, Whitfield GK, Hsieh JC, Thompson PD, Haussler CA and Haussler MR: Molecular nature of the vitamin D receptor and its role in regulation of gene expression. Rev Endocr Metabol Disord 2: 203-216, 2001.

14. Merza M, Farnia P, Anoosheh S, et al: The NRAMPI, VDR and TNF-alpha gene polymorphisms in Iranian tuberculosis patients: the study on host susceptibility. Braz J Infect Dis 13: 252-256, 2009.

15. Bellamy R, Beyers N, McAdam KP, et al: Genetic susceptibility to tuberculosis in Africans: a genome-wide scan. Proc Nat Acad Sci USA 97: 8005-8009, 2000.

16. Rebbeck TR, Jaffe JM, Walker AH, Wein AJ and Malkowicz SB: Modification of clinical presentation of prostate tumors by a novel genetic variant in CYP3A4. J Natl Cancer Inst 90: 1225-1229, 1998. 
17. Huang Z, Fasco MJ, Figge HL, Keyomarsi K and Kaminsky LS Expression of cytochromes P450 in human breast tissue and tumors. Drug Metab Dispos 24: 899-905, 1996.

18. Kolars JC, Lown KS, Schmiedlin-Ren P, et al: CYP3A gene expression in human gut epithelium. Pharmacogenetics 4 247-259, 1994

19. Lown KS, Bailey DG, Fontana RJ, et al: Grapefruit juice increases felodipine oral availability in humans by decreasing intestinal CYP3A protein expression. J Clin Invest 99: 2545-2553, 1997.

20. Shimada T, Yamazaki H, Mimura M, Inui Y and Guengerich FP Interindividual variations in human liver cytochrome P-450 enzymes involved in the oxidation of drugs, carcinogens and toxic chemicals: studies with liver microsomes of 30 Japanese and 30 Caucasians. J Pharmacol Exp Ther 270: 414-423, 1994.

21. Zheng W, Jin F, Dunning LA, et al: Epidemiological study of urinary 6beta-hydroxycortisol to cortisol ratios and breast cancer risk. Cancer Epidemiol Biomarkers Prev 10: 237-242, 2001.

22. Hashimoto H, Toide K, Kitamura R, et al: Gene structure of CYP3A4, an adult-specific form of cytochrome P450 in human livers, and its transcriptional control. Eur J Biochem 218: 585-595, 1993.

23. Itoh $\mathrm{S}$, Yanagimoto $\mathrm{T}$, Tagawa $\mathrm{S}$, et al: Genomic organization of human fetal specific P-450IIIA7 (cytochrome P-450HFLa)related gene(s) and interaction of transcriptional regulatory factor with its DNA element in the 5' flanking region. Biochim Biophys Acta 1130: 133-138, 1992.

24. Kinirons MT, Lang CC, He HB, et al: Triazolam pharmacokinetics and pharmacodynamics in Caucasians and Southern Asians: ethnicity and CYP3A activity. Br J Clin Pharmacol 41: 69-72, 1996.

25. Westlind A, Lofberg L, Tindberg N, Andersson TB and Ingelman-Sundberg M: Interindividual differences in hepatic expression of CYP3A4: relationship to genetic polymorphism in the 5'-upstream regulatory region. Biochem Biophys Res Commun 259: 201-205, 1999.

26. Sata F, Sapone A, Elizondo G, et al: CYP3A4 allelic variants with amino acid substitutions in exons 7 and 12: evidence for an allelic variant with altered catalytic activity. Clin Pharmacol Ther 67: 48-56, 2000

27. Plummer SJ, Conti DV, Paris PL, Curran AP, Casey G and Witte JS: CYP3A4 and CYP3A5 genotypes, haplotypes, and risk of prostate cancer. Cancer Epidemiol Biomarkers Prev 12: 928-932, 2003

28. Kadlubar FF, Berkowitz GS, Delongchamp RR, et al: The $C Y P 3 A 4^{*} 1 \mathrm{~B}$ variant is related to the onset of puberty, a known risk factor for the development of breast cancer. Cancer Epidemiol Biomarkers Prev 12: 327-331, 2003.

29. Spurdle AB, Goodwin B, Hodgson E, et al: The CYP3A4*1B polymorphism has no functional significance and is not associated with risk of breast or ovarian cancer. Pharmacogenetics 12 355-366, 2002.

30. Ministério da Saúde: Manual de Recomendações para o Controle da Tuberculose no Brasil. Secretaria de Vigilância em Saúde Programa Nacional de Controle da Tuberculose, 2010.

31. Ensembl: ensembl genomics.org. 2009.

32. ncbi: National Center for Biotechnology Information.

33. HGVS: Human Genome Variantion Society.

34. Keshava C, McCanlies EC and Weston A: CYP3A4 polymorphisms - potential risk factors for breast and prostate cancer: a HuGE review. Am J Epidemiol 160: 825-841, 2004.
35. aids.gov.br: Ministério da Saúde. Departamento de DST, Aids e Hepatites Virais.

36. Hirota T, Ieiri I, Takane H, et al: Allelic expression imbalance of the human CYP3A4 gene and individual phenotypic status. Hum Mol Genet 13: 2959-2969, 2004.

37. Slama K, Chiang CY, Enarson DA, et al: Tobacco and tuberculosis: a qualitative systematic review and meta-analysis. Int J Tuberc Lung Dis 11: 1049-1061, 2007.

38. Chiang CY, Slama K and Enarson DA: Associations between tobacco and tuberculosis. Int J Tuberc Lung Dis 11: 258-262, 2007.

39. Iglesias R, Jha P, Pinto M, Costa e Silva LV and Godinho J: Tobacco control in Brazil. In: HNP - Health, Nutrition and Population. The World Bank, p120, 2007.

40. Zeigler-Johnson CM, Walker AH, Mancke B, et al: Ethnic differences in the frequency of prostate cancer susceptibility alleles at SRD5A2 and CYP3A4. Hum Hered 54: 13-21, 2002.

41. Walker AH, Jaffe JM, Gunasegaram S, et al: Characterization of an allelic variant in the nifedipine-specific element of CYP3A4 ethnic distribution and implications for prostate cancer risk. Mutations in brief no. 191. Online. Human Mutat 12: 289, 1998.

42. Loukola A, Chadha M, Penn SG, et al: Comprehensive evaluation of the association between prostate cancer and genotypes/ haplotypes in CYP17A1, CYP3A4, and SRD5A2. Eur J Hum Genet 12: 321-332, 2004.

43. Fiegenbaum M, da Silveira FR, van der Sand CR, et al: The role of common variants of ABCB1, CYP3A4, and CYP3A5 genes in lipid-lowering efficacy and safety of simvastatin treatment. Clin Pharmacol Ther 78: 551-558, 2005.

44. Almeida S, Zandona MR, Franken N, Callegari-Jacques SM, Osorio-Wender MC and Hutz MH: Estrogen-metabolizing gene polymorphisms and lipid levels in women with different hormonal status. Pharmacogenom J 5: 346-351, 2005.

45. Alves-Silva J, da Silva Santos M, Guimaraes PE, et al: The ancestry of Brazilian mtDNA lineages. Am J Hum Genet 67: 444-461, 2000.

46. Schirmer M, Toliat MR, Haberl M, et al: Genetic signature consistent with selection against the CYP3A4*1B allele in nonAfrican populations. Pharmacogenet Genom 16: 59-71, 2006.

47. Felix CA, Walker AH, Lange BJ, et al: Association of CYP3A4 genotype with treatment-related leukemia. Proc Natl Acad Sci USA 95: 13176-13181, 1998.

48. Ando Y, Tateishi T, Sekido Y, et al: Re: Modification of clinical presentation of prostate tumors by a novel genetic variant in CYP3A4. J Nat Cancer Inst 91: 1587-1590, 1999.

49. Liu PT, Stenger S, Tang DH and Modlin RL: Cutting edge: vitamin D-mediated human antimicrobial activity against Mycobacterium tuberculosis is dependent on the induction of cathelicidin. J Immunol 179: 2060-2063, 2007.

50. Ho-Pham LT, Nguyen ND, Nguyen TT, et al: Association between vitamin D insufficiency and tuberculosis in a Vietnamese population. BMC Infect Dis 10: 306, 2010.

51. Wilkinson RJ, Llewelyn M, Toossi Z, et al: Influence of vitamin $\mathrm{D}$ deficiency and vitamin $\mathrm{D}$ receptor polymorphisms on tuberculosis among Gujarati Asians in west London: a casecontrol study. Lancet 355: 618-621, 2000. 